\title{
Effect of the Thickness of High Tc Superconducting Rectangular Microstrip Patch Over Ground Plane with Rectangular Aperture
}

\author{
Nabil Boukhennoufa ${ }^{1}$, Lotfi Djouane ${ }^{2}$, Houcine Oudira $^{3}$, Mounir Amir $^{4}$, Tarek Fortaki ${ }^{5}$ \\ ${ }^{1,2,3}$ Department of Electronics, University of Mohamed Boudiaf, M'sila, Algeria \\ ${ }^{1,2,3}$ Laboratory of Electrical Engineering, University of Mohamed Boudiaf, M'sila, Algeria \\ ${ }^{4}$ Scientific and Technical Research Center in Welding and Control, Algiers, Algeria \\ ${ }^{5}$ Department of Electronics, University of Mostefa Ben Boulaid, Batna, Algeria
}

\section{Article Info \\ Article history: \\ Received Jan 29, 2018 \\ Revised Apr 12, 2018 \\ Accepted Apr 18, 2018 \\ Keyword: \\ Full-wave analysis High Tc superconducting Microstrip patch Rectangular aperture}

\begin{abstract}
In recent years, a great interest has been observed in the development and use of new materials in microwave technology. Particularly, a special interest has been observed in the use of superconducting materials in microwave integrated circuits, this is due to their main characteristics. In this paper, the complex resonant frequency problem of a superconductor patch over Ground Plane with Rectangular Aperture is formulated in terms of an integral equation, the kernel of which is the dyadic Green's function. Galerkin's procedure is used in the resolution of the electric field integral equation. The surface impedance of the superconductor film is modeled using the two fluids model of Gorter and Casimir. Numerical results concerning the effect of the thickness of the superconductor patch on the characteristics of the antenna are presented.
\end{abstract}

Copyright ( 2018 Institute of Advanced Engineering and Science. All rights reserved.

\section{Corresponding Author:}

Nabil Boukhennoufa,

Department of Electronics,

Laboratory of Electrical Engineering,

University of Mohamed Boudiaf, M'sila,

BP 166 M'sila 28000, Algeria.

Email: boukhennoufa.nabil@gmail.com

\section{INTRODUCTION}

Microwave passive devices such as resonators, filters and antennas are one of the main applications of the high TC superconducting (HTS) materials. They show superior performance and characteristics to the normal metal devices, such as: the power losses are low, reduction of attenuation and noise level, besides the propagation time of signals in the circuit can be greatly reduced [1]-[6]. This allows to be used in several applications such as mobile communications, radars and filters [7]-[10]. The study of the resonant characteristics of High Tc superconducting microstrip antennas has been first studied by [4] using the cavity model. Since the cavity model does not consider rigorously the effects of surface waves and fringing fields at the edge of the patch. Recently; Silva has studied the resonant characteristics of High Tc superconducting microstrip antennas using the full-wave analysis [11], [12]. It is noted that in the above works, the effect of thin superconducting patch was not studied.

In this paper, the effect of thin superconducting patch loading on the resonant frequency and bandwidth of rectangular microstrip structures illustrated in Figure 1 is investigated. The complex resonant frequency problem considered here is formulated in terms of an integral equation using vector Fourier transforms [13]. The surface impedance of the superconductor film is modeled using the two fluids model of Gorter and Casimir [5], [11], [14]. The paper is organized as follows. First, the integral equation for the unknown patch currents is formulated. The derivation is performed in the Fourier transform domain and 
utilized the dyadic Green's function of the considered structure, without taking into account the effect of the superconductivity. To include the effect of the superconductivity of the patch, surface complex impedance, $\mathrm{Zs}$ is considered. The Galerkin moment method is used to solve the integral equation. The characteristic equation for the complex resonant frequencies is given. Various numerical results are given in Section 3. Finally, conclusions are summarized in Section 4.

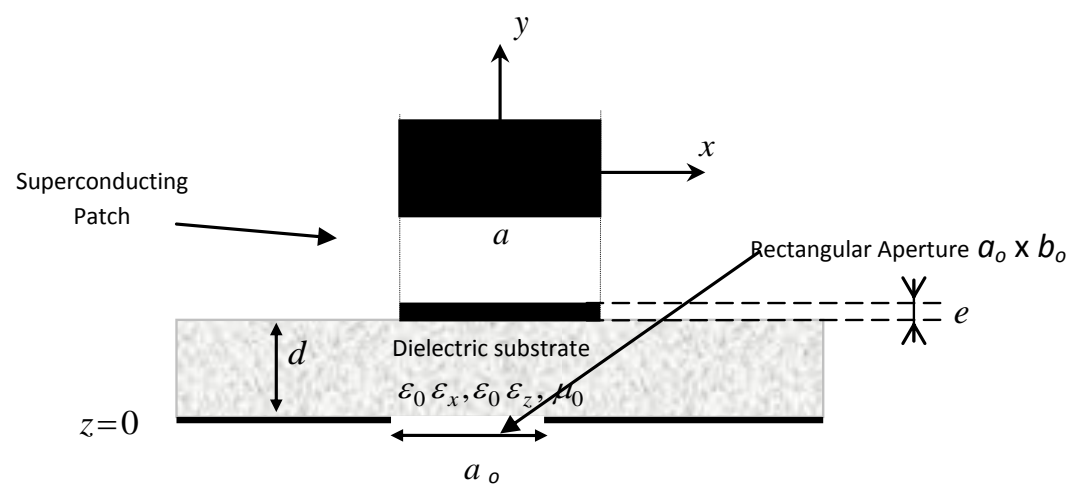

Figure 1. Geometrical structure of a superconducting rectangular microstrip patch

\section{ANALYSIS METHOD}

In this paper an efficient technique to derive the dyadic Green's functions for Superconducting microstrip patch over ground plane with aperture is proposed. The transverse field inside isotropic region $(0<z<d)$ can be obtained via the inverse vector Fourier transforms as:

$$
\begin{gathered}
\boldsymbol{E}\left(\boldsymbol{r}_{s}, z\right)=\frac{1}{4 \pi^{2}} \int_{-\infty}^{+\infty} \int_{-\infty}^{+\infty} \overline{\boldsymbol{F}}\left(\boldsymbol{k}_{s}, \boldsymbol{r}_{s}\right) \cdot \boldsymbol{e}\left(\boldsymbol{k}_{s}, z\right) d k_{x} d k_{y} \\
\boldsymbol{H}\left(\boldsymbol{r}_{s}, z\right)=\frac{1}{4 \pi^{2}} \int_{-\infty}^{+\infty} \int_{-\infty}^{+\infty} \overline{\boldsymbol{F}}\left(\boldsymbol{k}_{s}, \boldsymbol{r}_{s}\right) \boldsymbol{h}\left(\boldsymbol{k}_{s}, z\right) d k_{x} d k_{y}
\end{gathered}
$$

Where: $\overline{\boldsymbol{F}}\left(\boldsymbol{k}_{s}, \boldsymbol{r}_{s}\right)=\frac{1}{k_{s}}\left[\begin{array}{cc}k_{x} & k_{y} \\ k_{y} & -k_{x}\end{array}\right] e^{i \boldsymbol{k}_{s} \cdot \boldsymbol{r}_{s}}, \boldsymbol{r}_{s}=\hat{\boldsymbol{x}} x+\hat{\boldsymbol{y}} y, \boldsymbol{k}_{s}=\hat{\boldsymbol{x}} k_{x}+\hat{\boldsymbol{y}} k_{y}, k_{s}=\left|\boldsymbol{k}_{s}\right|$.

$$
\begin{aligned}
& \boldsymbol{e}\left(\boldsymbol{k}_{s}, z\right)=e^{-i \overline{\boldsymbol{k}}_{z} z} \cdot \boldsymbol{A}\left(\boldsymbol{k}_{s}\right)+e^{i \overline{\boldsymbol{k}}_{z} z} \cdot \boldsymbol{B}\left(\boldsymbol{k}_{s}\right) \\
& \boldsymbol{h}\left(\boldsymbol{k}_{s}, z\right)=\overline{\boldsymbol{g}}\left(\boldsymbol{k}_{s}\right) \cdot\left[e^{-i \overline{\boldsymbol{k}}_{z} z} \cdot \boldsymbol{A}\left(\boldsymbol{k}_{s}\right)-e^{i \overline{\boldsymbol{k}}_{z} z} \cdot \boldsymbol{B}\left(\boldsymbol{k}_{s}\right)\right]
\end{aligned}
$$

In (3) and (4), $A$ and $B$ are to component unknown vectors and

$$
\overline{\boldsymbol{k}}_{z}=\operatorname{diag}\left[k_{z}^{e}, k_{z}^{h}\right], \overline{\boldsymbol{g}}\left(\boldsymbol{k}_{s}\right)=\operatorname{diag}\left[\frac{\omega \varepsilon_{0} \varepsilon_{x}}{k_{z}^{e}}, \frac{k_{z}^{h}}{\omega \mu_{0}}\right]
$$

$k_{z}^{e}$ and $k_{z}^{h}$ are propagation constants for TM and TE waves respectively in substrate [15]-[18]. Writing (3) and (4) in the plane $z=0$ and $z=d$, and by eliminating the unknowns $A$ and $B$, we obtain the matrix form:

$$
\left[\begin{array}{l}
\boldsymbol{e}\left(\boldsymbol{k}_{s}, d^{-}\right) \\
\boldsymbol{h}\left(\boldsymbol{k}_{s}, d^{-}\right)
\end{array}\right]=\overline{\boldsymbol{T}} \cdot\left[\begin{array}{l}
\boldsymbol{e}\left(\boldsymbol{k}_{s}, O^{+}\right) \\
\boldsymbol{h}\left(\boldsymbol{k}_{s}, O^{+}\right)
\end{array}\right]
$$


With

$$
\overline{\boldsymbol{T}}=\left[\begin{array}{ll}
\overline{\boldsymbol{T}}^{11} & \overline{\boldsymbol{T}}^{12} \\
\overline{\boldsymbol{T}}^{21} & \overline{\boldsymbol{T}}^{22}
\end{array}\right]=\left[\begin{array}{cc}
\cos \overline{\boldsymbol{\theta}} & -i \overline{\boldsymbol{g}}^{-1} \cdot \sin \overline{\boldsymbol{\theta}} \\
-i \overline{\boldsymbol{g}} \cdot \sin \overline{\boldsymbol{\theta}} & \cos \overline{\boldsymbol{\theta}}
\end{array}\right], \overline{\boldsymbol{\theta}}=\overline{\boldsymbol{k}}_{z} d
$$

Equation (7) combines $e$ and $h$ on both sides of substrate as input and output quantities. The continuity equations for the tangential field components at the interface $z=d$ are:

$$
\begin{aligned}
& \boldsymbol{e}\left(\boldsymbol{k}_{s}, d^{-}\right)=\boldsymbol{e}\left(\boldsymbol{k}_{s}, d^{+}\right)=\boldsymbol{e}\left(\boldsymbol{k}_{s}, d\right)=\boldsymbol{e}\left(\boldsymbol{k}_{s}\right) \\
& \boldsymbol{h}\left(\boldsymbol{k}_{s}, d^{-}\right)-\boldsymbol{h}\left(\boldsymbol{k}_{s}, d^{+}\right)=\boldsymbol{j}\left(\boldsymbol{k}_{s}\right)
\end{aligned}
$$

$\boldsymbol{j}\left(\boldsymbol{k}_{s}\right)$ in (9) is related to the vector Fourier transform of $\boldsymbol{J}\left(\boldsymbol{r}_{s}\right)$, the current on the patch, as [19]

$$
\boldsymbol{j}\left(\boldsymbol{k}_{s}\right)=\int_{-\infty}^{+\infty} \int_{-\infty}^{+\infty} \overline{\boldsymbol{F}}\left(\boldsymbol{k}_{s},-\boldsymbol{r}_{s}\right) \boldsymbol{J}\left(\boldsymbol{r}_{s}\right) d k_{x} d k_{y}, \boldsymbol{J}\left(\boldsymbol{r}_{s}\right)=\left[\begin{array}{l}
J_{x}\left(\boldsymbol{r}_{s}\right) \\
J_{y}\left(\boldsymbol{r}_{s}\right)
\end{array}\right]
$$

The continuity equations for the tangential field components at $z=0$ are:

$$
\begin{aligned}
& \boldsymbol{e}\left(\boldsymbol{k}_{s}, O^{-}\right)=\boldsymbol{e}\left(\boldsymbol{k}_{s}, O^{+}\right)=\boldsymbol{e}\left(\boldsymbol{k}_{s}, O\right)=\boldsymbol{e}_{0}\left(\boldsymbol{k}_{s}\right) \\
& \boldsymbol{h}\left(\boldsymbol{k}_{s}, O^{-}\right)-\boldsymbol{h}\left(\boldsymbol{k}_{s}, O^{+}\right)=\boldsymbol{j}_{0}\left(\boldsymbol{k}_{s}\right)
\end{aligned}
$$

In (12), $\boldsymbol{j}_{0}\left(\boldsymbol{k}_{s}\right)$ is the vector Fourier transform of the current $\boldsymbol{J}_{0}\left(\boldsymbol{r}_{s}\right)$ on the ground plane with a rectangular aperture. In the unbounded air region above the patch the electromagnetic field given by (3) and (4) should at $z \rightarrow+\infty(z \rightarrow-\infty)$ according to Summerfield's condition of radiation, and this yields:

$$
\begin{aligned}
& \boldsymbol{h}\left(\boldsymbol{k}_{s}, d^{+}\right)=\overline{\boldsymbol{g}}_{0}\left(\boldsymbol{k}_{s}\right) \cdot \boldsymbol{e}\left(\boldsymbol{k}_{s}, d^{+}\right) \\
& \boldsymbol{h}\left(\boldsymbol{k}_{s}, O^{-}\right)=-\overline{\boldsymbol{g}}_{O}\left(\boldsymbol{k}_{s}\right) \cdot \boldsymbol{e}\left(\boldsymbol{k}_{s}, O^{-}\right)
\end{aligned}
$$

Where $\overline{\boldsymbol{g}}_{0}\left(\boldsymbol{k}_{s}\right)$ can be easily obtained from the expression of $\overline{\boldsymbol{g}}\left(\boldsymbol{k}_{s}\right)$ given in (5) by allowing $\varepsilon_{x}=\varepsilon_{z}=\varepsilon_{r}=1$. Combining (6), (8), (9) and (1)-(14), we obtain a relation among $\boldsymbol{j}\left(\boldsymbol{k}_{s}\right), \boldsymbol{j}\left(\boldsymbol{k}_{s}\right), \boldsymbol{e}\left(\boldsymbol{k}_{s}\right)$ and $\boldsymbol{e}_{0}\left(\boldsymbol{k}_{\boldsymbol{s}}\right)$ given by:

$$
\begin{aligned}
& {\left[\begin{array}{c}
\tilde{E}_{x} \\
\tilde{E}_{y}
\end{array}\right]=\left[\begin{array}{ll}
Q_{x x} & Q_{x y} \\
Q_{y x} & Q_{y y}
\end{array}\right] \cdot\left[\begin{array}{c}
\tilde{J}_{x} \\
\tilde{J}_{y}
\end{array}\right]+\left[\begin{array}{ll}
W W_{x x} & W W_{x y} \\
W W_{y x} & W W_{y y}
\end{array}\right] \cdot\left[\begin{array}{c}
\tilde{E}_{x 0} \\
\tilde{E}_{y 0}
\end{array}\right]} \\
& {\left[\begin{array}{c}
\tilde{J}_{x 0} \\
\tilde{J}_{y 0}
\end{array}\right]=-\left[\begin{array}{ll}
W W_{x x} & W W_{x y} \\
W W_{y x} & W W_{y y}
\end{array}\right] \cdot\left[\begin{array}{c}
\tilde{J}_{x} \\
\tilde{J}_{y}
\end{array}\right]-\left[\begin{array}{cc}
Y_{x x} & Y_{x y} \\
y_{y x} & y_{y y}
\end{array}\right] \cdot\left[\begin{array}{c}
\tilde{E}_{x 0} \\
\tilde{E}_{y 0}
\end{array}\right]}
\end{aligned}
$$

To include the effect of the superconducting of the microstrip film, the dyadic Green's function is modified by considering a surface complex impedance $Z s$, is determinate by using the model of Gorter and Casimir [5], [11], [14].

$$
Z_{s}=\sqrt{\frac{\omega \mu_{0}}{2 \sigma}}
$$

If the thickness of the superconducting film $R$ is less than three penetration depths, a better boundary 
condition is given by [5]:

$$
Z_{s}=\frac{1}{R \cdot \sigma}
$$

Where the complex conductivity is given by [5], [7]

$$
\sigma=\sigma_{n}\left(\frac{T}{T_{C}}\right)^{4}+\left(\left(1-\left(\frac{T}{T_{C}}\right)^{4}\right) /\left(i \omega \mu_{0} \lambda_{0}^{2}\right)\right)
$$

With $\lambda_{0}$ is the skin depth at zero temperature, $\sigma_{\mathrm{n}}$ is the conductivity of normal electrons and $T_{c}$ critical temperature of the superconductor.

The electric field and the surface current densities total in the interface $z=d\left(\tilde{\mathrm{E}}_{T}, \tilde{\mathrm{J}}_{T}\right)$ are the electric fields and the surface current densities in the film $\left(\tilde{\mathrm{E}}_{T}^{i}, \tilde{\mathrm{J}}_{T}^{i}\right)$ and out the film $\left(\tilde{\mathrm{E}}_{T}^{O}, \tilde{\mathrm{J}}_{T}^{O}\right)$ respectively.

$$
\left\{\begin{array}{l}
\tilde{\boldsymbol{E}}_{T}=\tilde{\boldsymbol{E}}_{T}^{i}+\tilde{\boldsymbol{E}}_{T}^{o} \\
\tilde{\boldsymbol{J}}_{T}=\tilde{\boldsymbol{J}}_{T}^{i}+\tilde{\boldsymbol{J}}_{T}^{o}
\end{array}\right.
$$

By substituting Equation (20) in Equation (15), we obtain

$$
\begin{aligned}
& {\left[\begin{array}{c}
\tilde{E}_{x}{ }^{o} \\
\tilde{E}_{y}{ }^{o}
\end{array}\right]=\left[\begin{array}{cc}
Q_{x x}-Z_{s} & Q_{x y} \\
Q_{y x} & Q_{y y}-Z_{s}
\end{array}\right] \cdot\left[\begin{array}{c}
\tilde{J}_{x} \\
\tilde{J}_{y}
\end{array}\right]+\left[\begin{array}{ll}
W W_{x x} & W W_{x y} \\
W W_{y x} & W W_{y y}
\end{array}\right] \cdot\left[\begin{array}{c}
\tilde{E}_{x 0} \\
\tilde{E}_{y 0}
\end{array}\right]} \\
& {\left[\begin{array}{c}
\tilde{J}_{x 0} \\
\tilde{J}_{y 0}
\end{array}\right]=-\left[\begin{array}{ll}
W W_{x x} & W W_{x y} \\
W W_{y x} & W W_{y y}
\end{array}\right] \cdot\left[\begin{array}{c}
\tilde{J}_{x} \\
\tilde{J}_{y}
\end{array}\right]-\left[\begin{array}{cc}
Y_{x x} & Y_{x y} \\
y_{y x} & y_{y y}
\end{array}\right] \cdot\left[\begin{array}{c}
\tilde{E}_{x o} \\
\tilde{E}_{y 0}
\end{array}\right]}
\end{aligned}
$$

Boundary conditions require that the transverse electric field of (21) vanishes on the superconducting patch and the current of (22) varnishes off ground plane, to give the following coupled integral equations for the patch current and aperture field:

$$
\begin{aligned}
& \int_{-\infty}^{+\infty} \int_{-\infty}^{+\infty}\left[\left(Q_{x x}-Z_{s}\right) \tilde{J}_{x}+Q_{x y} \tilde{J}_{y}+W W_{x x} \tilde{E}_{x 0}+W W_{x y} \tilde{E}_{y 0}\right] \exp \left(i k_{x} x+i k_{y} y\right) d k_{x} d k_{y}=0 \\
& \int_{-\infty}^{+\infty} \int_{-\infty}^{+\infty}\left[Q_{y x} \tilde{J}_{x}+\left(Q_{y y}-Z_{s}\right) \tilde{J}_{y}+W W_{y x} \tilde{E}_{x 0}+W W_{y y} \tilde{E}_{y 0}\right] \exp \left(i k_{x} x+i k_{y} y\right) d k_{x} d k_{y}=0 \\
& \int_{-\infty}^{+\infty} \int_{-\infty}^{+\infty}\left[W W_{x x} \tilde{J}_{x}+W W_{x y} \tilde{J}_{y}+Y_{x x} \tilde{E}_{x 0}+Y_{x y} \tilde{E}_{y 0}\right] \exp \left(i k_{x} x+i k_{y} y\right) d k_{x} d k_{y}=0 \\
& \int_{-\infty}^{+\infty} \int_{-\infty}^{+\infty}\left[W W_{y x} \tilde{J}_{x}+W W_{y y} \tilde{J}_{y}+Y_{y x} \tilde{E}_{x o}+Y_{y y} \tilde{E}_{y 0}\right] \exp \left(i k_{x} x+i k_{y} y\right) d k_{x} d k_{y}=0
\end{aligned}
$$

\section{NUMERICAL RESULTS AND DISCUSSION}

Table 1 summarizes the calculated resonance frequencies and those obtained through the cavity model [6] for three different widths of the patch and differences between these results dues less than $2 \%$ are obtained. Excellent agreement between our results and those calculated by Full-wave analysis [11] and those obtained by Cavity Model [6] is observed and represented in Table 2. 
Table 1. Comparison of Calculated Resonant Frequencies with those Presented by Richard et al [6] $a=1630 \mu \mathrm{m}, \sigma_{n}=10^{6} \mathrm{~S} / \mathrm{M}, T_{c}=89 \mathrm{~K}, \lambda_{0}=140 \mathrm{~nm}, e=350 \mathrm{~nm} ; \mathrm{T}=77 \mathrm{~K}, \varepsilon_{r}=23.81, d=254 \mu \mathrm{m}$ and $a_{0}=0$

\begin{tabular}{cccc}
\hline$b(\mu \mathrm{m})$ & \multicolumn{2}{c}{ Resonance Frequencies $(\mathrm{GHz})$} & Error (\%) \\
\hline 935 & Cavity Model [6] & Our results & 0.66 \\
1050 & 28.95 & $\mathbf{2 8 . 7 6}$ & 0.65 \\
1100 & 26.12 & $\mathbf{2 6 . 2 9}$ & 1.12 \\
\hline
\end{tabular}

Table 2. Comparison of Calculated Resonant Frequencies with those Presented by Richard et al [6] and Silva et al [11] $a=1630 \mu \mathrm{m}, b=935 \mu \mathrm{m}, \sigma_{n}=10^{6} \mathrm{~S} / \mathrm{M}, T_{c}=89 \mathrm{~K}, \lambda_{0}=140 \mathrm{~nm}, e=350 \mathrm{~nm} ; \mathrm{T}=50 \mathrm{~K}, d=254 \mu \mathrm{m}$ and $a_{0}=0$

\begin{tabular}{cccc}
\hline Relative Permittivity & & Resonance Frequencies (GHz) & Our results \\
$\left(\varepsilon_{r}\right)$ & Full-wave analysis [11] & Cavity Model [6] & 41.585 \\
11 & 41.041 & 41.638 & 34.816 \\
23.81 & 34.856 & 35.300 & 28.764 \\
\hline
\end{tabular}

The influence of the thickness of the superconducting film on the operating frequency and bandwidth of the antenna without and with rectangular aperture is studied in Figure 2 and Figure 3. The thickness of the rectangular patch is normalized with respect to the penetration depth to zero temperature. In the case of the antenna having a rectangular aperture, the size thereof is $163 \mu \mathrm{m} \times 93.5 \mu \mathrm{m}$. The characteristics of the superconducting film are: $\lambda_{0}=100 \mathrm{~nm}, \sigma_{n}=9.8310^{5} \mathrm{~S} / \mathrm{M}$ and $T_{c}=89 \mathrm{~K}$. For the microstrip antenna, the following parameters are used: $a=1630 \mu \mathrm{m}, b=935 \mu \mathrm{m}, b=254 \mu \mathrm{m}$ and $\varepsilon_{r}=23.81$. The operating temperature is $T=50 \mathrm{~K}$.

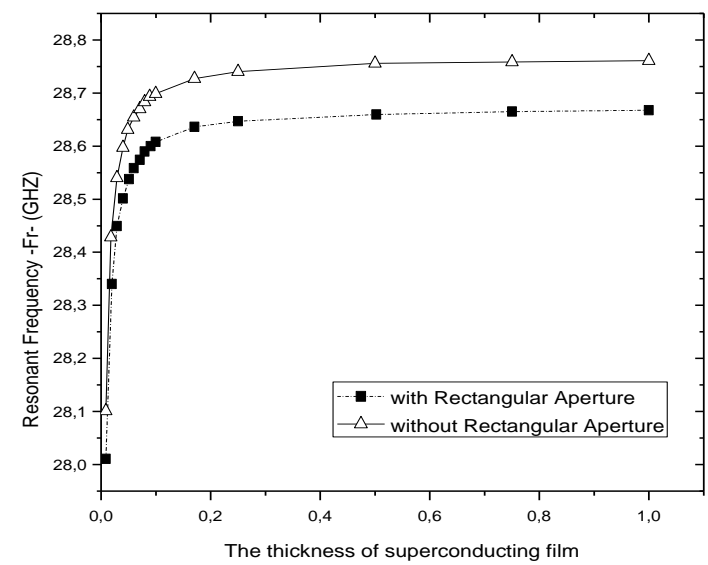

Figure 2. Resonant frequency of a superconducting microstrip antenna with and without aperture in the ground plane according to the standardized thickness of the superconducting film

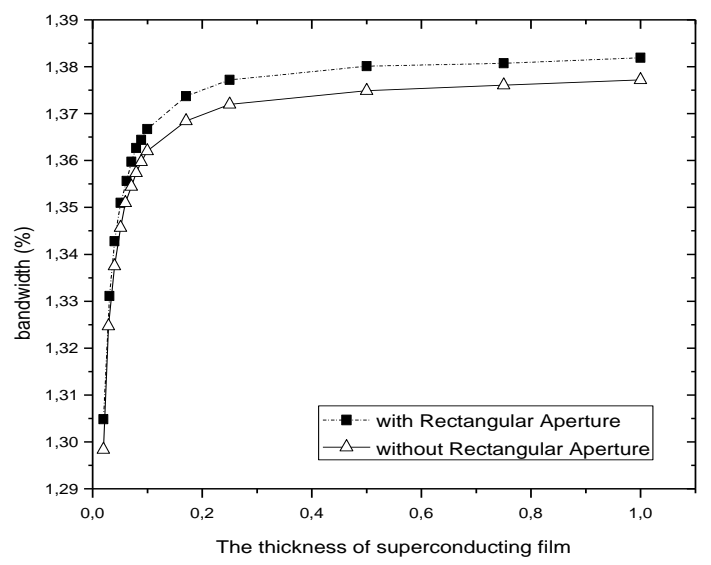

Figure 3. Bandwidth of a superconducting microstrip antenna with and without aperture in the ground plane according to the standardized thickness of the superconducting film

It is noted that when the thickness of the superconductor film increases, the resonant frequency and bandwidth of the antenna without increasing opening as well as to the antenna aperture. Note that the effect of the thickness of the superconducting film is larger for small values of $e\left(e<0.1 \lambda_{0}\right)$. When $e$ exceeds $0.1 \lambda_{0}$, the increase in the thickness of the superconducting film slowly increases the resonant frequency and bandwidth. Extreme care should be taken when designing a microstrip antenna with a thin superconducting film, since a small uncertainty when the patch is made may result in a significant gap in the frequency and bandwidth of the antenna.

Figure 4 plots the radiation patterns in the $E$-plane $(\phi=0)$ and $H$-plane $(\phi=\pi / 2)$ of a rectangular microstrip patch over the ground planes with and without rectangular apertures in both the air half-space above the patch and the concerning radiation above the patch. It is clear that it decreases with the increase of the size of the aperture. 

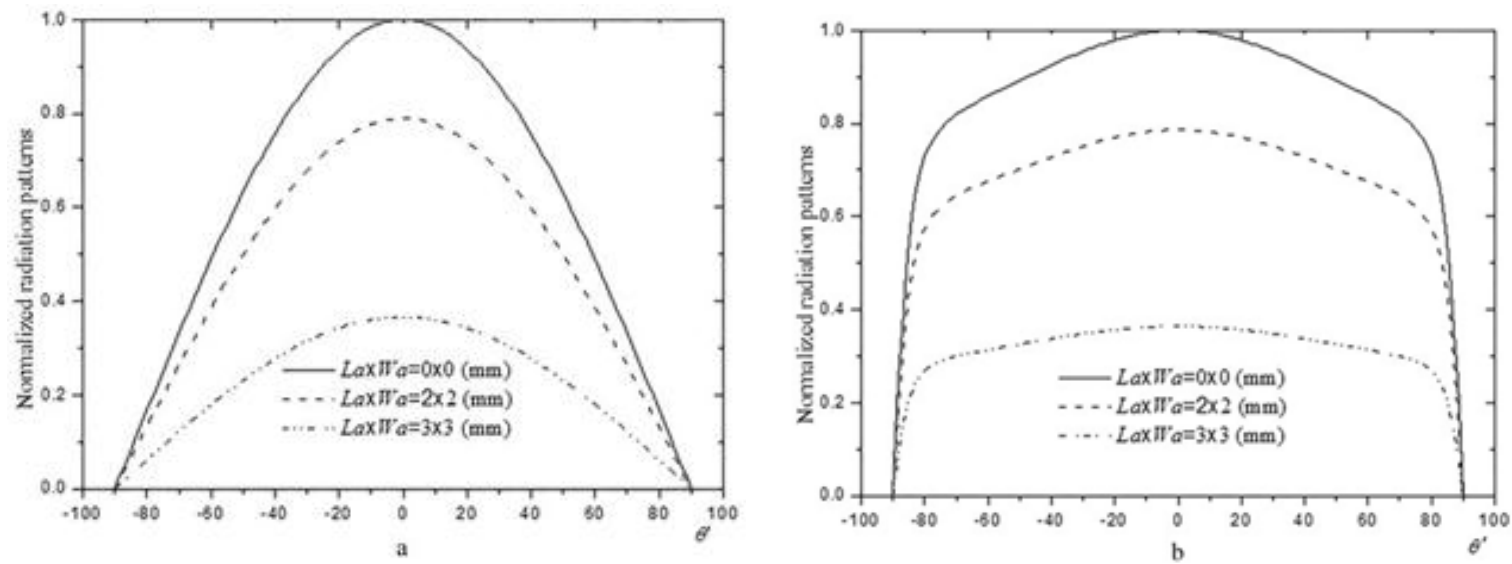

Figure 4. Radiation patterns of rectangular patches over the ground planes with and without rectangular apertures (a) $H$-plane, (b) $E$-plane ; with $L_{p}=15 \mathrm{~mm}, W_{p}=10 \mathrm{~mm}, \varepsilon_{r}=7, d=1 \mathrm{~mm} \lambda_{0}=100 \mathrm{~nm}, \sigma_{n}=9.8310^{5} \mathrm{~S} / \mathrm{M}$ and $T_{c}=89 \mathrm{~K}$

\section{CONCLUSION}

We have presented a rigorous full-wave analysis of rectangular microstrip patch over Ground Plane with Rectangular Aperture using superconducting materials. The problem has been formulated in terms of integral equations using vector Fourier transforms. An efficient technique has been used for determining the dyadic Green's functions. Galerkin's method has been used to solve the surface current density on the rectangular patch. The calculated results have been compared with calculated and measured ones available in the literature and excellent agreement has been found.

Extreme care should be taken when designing a microstrip antenna with thin superconducting patch; since small uncertainty in thickness of the superconducting patch can result in an important detuning of the frequency.

\section{REFERENCES}

[1] S. Bedra, T. Fortaki, A. Messai and R. Bedra, "Spectral Domain Analysis of Resonant Characteristics of High Tc Superconducting Rectangular Microstrip Patch Printed on Isotropic or Uniaxial Anisotropic Substrates", Wireless Personal Communications, vol. 86, no 2, pp. 495-511, 2016.

[2] F. Benmeddour, C. Dumond, F. Benabdelaziz and F. Bouttout, "Improving the performances of a high Tc superconducting circular microstrip antenna with multilayered configuration and anisotropic dielectrics", Progress In Electromagnetics Research C, vol. 18, pp. 169-183, 2011.

[3] N. Sekiya, A. Kubota, A. Kondo, S. Hirano, A. Saito and S. Ohshima, "Broadband superconducting microstrip patch antenna using additional gap-coupled resonators", Physica C, pp. 445-448, 2006.

[4] A. Cassinese, M. Barra, I. Fragala, M. Kusunoki, G. Malandrino, T. Nakagawa, L.M.S. Perdicaro, K. Sato, S. Ohshima and R. Vaglio, "Superconducting antennas for telecommunication applications based on dual mode cross slotted patches", Physica C, pp. 372-376, 2002.

[5] B.B.G. Klopman and H. Rogalla, "The propagation characteristics of wave-guiding structures with very thin superconductors; Application to coplanar waveguide YBa2 CU3O7-x resonators", IEEE Trans. Microwave Theory Tech., vol. 41, no. 5, pp. 781-791, 1993.

[6] M.A. Richard, K.B. Bhasin and P.C. Clapsy, "Superconducting microstrip antennas: An experimental comparison of two feeding methods", IEEE Trans. Antennas Propagat., Vol. 41, No 7, pp. 967-974, 1993.

[7] T. Firmansyah, Herudin, Suhendar, R. Wiryadinata, M. Iman Santoso, Y. R. Denny and T. Supriyanto, "Bandwidth and Gain Enhancement of MIMO Antenna by Using Ring and Circular Parasitic with Air-Gap Microstrip Structure", TELKOMNIKA (Telecommunication Computing Electronics and Control), vol. 15, no .3, pp. 1155-1163, 2017.

[8] A. Singh, Kamakshi, M. Aneesh and J.A. Ansari. "Slots and Notches Loaded Microstrip Patch Antenna for Wireless Communication”, TELKOMNIKA (Telecommunication Computing Electronics and Control), vol. 13, no 3, pp. 584-594, 2015.

[9] D. Fitsum, D. Mali and M. Ismail, "Dual-Band Proximity Coupled Feed Microstrip Patch Antenna with 'T' Slot on the Radiating Patch and 'Dumbbell' Shaped Defected Ground Structure”, Indonesian Journal of Electrical Engineering and Computer Science, vol. 3, no. 2, pp. 435-440, 2016. 
[10] D. Fistum, D. Mali and M. Ismail, "Bandwidth Enhancement of Rectangular Microstrip Patch Antenna using Defected Ground Structure", Indonesian Journal of Electrical Engineering and Computer Science, vol. 3, no. 2, pp. 428-434, 2016.

[11] S.G. da Silva, A.G. d'Assuçäo and J.R.S. Oliveira, "Analysis of high Tc superconducting microstrip antennas and arrays", SBMO/IEEE MTT-S IMOC., 1999.

[12] T. Fortaki, D. Khedrouche, F. Bouttout and A. Benghalia, "Vector Hankel transform analysis of a tunable circular microstrip patch", Commun. Numer. Meth. Engng, pp. 219-231, 2005.

[13] T. Itoh, "A full-wave analysis method for open microstrip structures", IEEE Trans. Antennas Propagat., vol. 29, no. 1, pp. 63-67, 1981.

[14] Z. Cai and J. Bornemann, "Generalized spectral-domain analysis for multilayered complex media and high-Tc superconductor applications", IEEE Trans. Microwave Theory Tech., vol. 40, no. 12, pp. 2251-2257, 1992.

[15] T. Fortaki and A. Benghalia, "Rigorous full-wave analysis of rectangular microstrip patches over ground planes with rectangular apertures in multilayered substrates that contain isotropic and uniaxial anisotropic materials", Microwave Opt. Technol. Lett., vol. 41, no. 6, pp. 496-500, 2004.

[16] T. Fortaki, D. Khedrouche, F. Bouttout, and A. Benghalia, "Numerical analysis of rectangular microstrip patch over ground plane with rectangular aperture", Commun. Numer. Meth. Engng. (John Wiley \& Sons), vol. 20, no. 6, pp. 489-500, 2004.

[17] T. Fortaki. and A. Benghalia, "Study of rectangular microstrip patch over ground plane with rectangular aperture in the presence of a high-permittivity dielectric layer below the aperture", Proceeding of the 16th International Conference on Microelectronics, Tunis, Tunisia, December 2004.

[18] C. Zebiri M. Lashab and F. Benabdelaziz, "Effect of anisotropic magneto-chirality on the characteristics of a microstrip resonator", IET Microw. Antennas Propag., vol. 4, no. 4, pp. 446-452, 2010.

[19] L. Djouane, S. Bedra, R. Bedra and T. Fortaki, "Neurospectral modeling of rectangular patch with rectangular aperture in the ground plane", International Journal of Microwave and Wireless Technologies, vol. 7, no. 6, pp. 759-768, 2015. 